\title{
Klebsiella pneumoniae necrotizing fasciitis on the upper lip in a patient with uncontrolled diabetes
}

\author{
Hyeong Seop Kim, \\ Yong Joon Chang, \\ Chul Hoon Chung \\ Department of Plastic and \\ Reconstructive Surgery, Kangdong \\ Sacred Heart Hospital, Seoul, Korea
}

\begin{abstract}
A 60-year-old woman with a history of diabetes mellitus and chronic renal failure was admitted to the hospital with severe pain in the upper lip, which began 4 days prior to admission, accompanied by a bullous lesion and suspected cellulitis in the upper lip. Immediately after admission, as the patient's general condition worsened, tests revealed a non-ST elevated myocardial infarction, septic embolism of the lung, as well as septic shock. Her upper lip suddenly presented a gangrenous and necrotic change, which the tissue and blood culture confirmed to be a Klebsiella pneumoniae infection. After a quick response, the patient's general condition improved. Subsequently, serial debridement was performed to effectively clear away the purulent discharge. While under general anesthesia, the process confirmed full-layer necrosis of the upper lip including the orbicularis oris muscle. Almost half of the entire upper lip sustained a full-layer skin and soft tissue defect, with scar contracture. Six months later, to correct the drooling and lip sealing following the defects, a scar release and an Abbe flap coverage were performed considering both functional and aesthetic aspects. The follow-up revealed a favorable corrective result of the upper lip drooling, and the patient was satisfied from a functional perspective.
\end{abstract}

Keywords: Fasciitis, necrotizing / Klebsiella pneumoniae / Lips / Pedicled flap / Reconstructive surgery / Shock, septic

\section{INTRODUCTION}

Necrotizing fasciitis (NF) is a life-threatening infection that extensively invades the fascia and subcutaneous layer. NF in the head and neck region comprises approximately $2.6 \%$ to $5 \%$ of total NF cases [1,2]. The scarce reports and low incidence may be due to the high vascularity of these regions $[3,4]$. NF of the head and neck is commonly of odontogenic origin and, due to its adjacency to the oral cavity, the lip is highly susceptible [5-7]. The most common pathogen causing NF has been reported as

Correspondence: Yong Joon Chang

Department of Plastic and Reconstructive Surgery, Kangdong Sacred Heart

Hospital, 150 Seongan-ro, Gangdong-gu, Seoul 05355, Korea

E-mail: drjunys@naver.com

This article was presented at the PRS Korea 2019 on November 9, 2019 in Seoul, Korea. Received November 19, 2019 / Revised March 12, 2020 / Accepted March 31, 2020 group A streptococcus (GAS); however, recently, there have been increasing reports of Klebsiella pneumoniae as another significant pathogen, with its rapid and aggressive features accentuating its clinical significance $[8,9]$. In this study, we elucidate our experience with treating NF in the upper lip of a patient with uncontrolled diabetes mellitus (DM). There was a high risk of mortality due to non-ST elevated myocardial infarction (NSTEMI) and the positive tissue culture for K. pneumoniae obtained immediately after admission, which led to rapid worsening of the disease and progression to septic shock. Nevertheless, due to intensive medical management and the use of intravenous broad-spectrum antibiotics, accompanied by active debridement and subsequent reconstruction, the interventions for this case were successful. 


\section{CASE REPORT}

A 60-year-old woman with a history of DM and chronic renal failure was admitted to the hospital with severe pain in her upper lip which began 4 days earlier, with a bullous lesion and suspected cellulitis in the upper lip. A special trauma history was denied. Since a zoster infection was suspected, she was admitted to the department of dermatology where antiviral treatment was initiated. Although she was aware of having DM, she did not regularly adhere to her medication. At the time of admission, she had a serum glucose level of $346 \mathrm{mg} / \mathrm{dL}$, hemoglobin Alc of $7.8 \%$, white blood cell count of $12,000 / \mu \mathrm{L}$, and C-reactive protein level of $93.7 \mathrm{mg} / \mathrm{L}$.

At midnight on the 1st day of admission, she complained of sudden chest pain; and electrocardiography and cardiac enzyme measurements confirmed the diagnosis of NSTEMI. Consequently, she was moved to the intensive care unit and transferred to the department of internal medicine. During the night, a sudden high fever exceeding $38^{\circ} \mathrm{C}$ and hypotension were observed, mental status deteriorated to stupor, and her general condition worsened. The swelling and inflammation of her upper lip increased, and later, an ulcerative lesion on the oral mucosa of the upper lip was detected. The intra-oral wound had a foul odor with purulent discharge. Laboratory reports showed a white blood cell count of $23,000 / \mu \mathrm{L}$ and a C-reactive protein level of $385.8 \mathrm{mg} / \mathrm{L}$ which strongly suggested septic shock, and warranted work-ups such as blood and pus culture tests. Coronary angiography performed following the NSTEMI confirmed a 3-vessel disease, with severe stenosis that made stent treatment virtually impossible. Due to the patient's immediate mortality risk, intravenous broad-spectrum antibiotics, boosters, and other adjunctive treatment measures were commenced immediately.

On the 2nd day of admission, the upper lip gangrene and necrosis rapidly progressed (Fig. 1A). Contrast-enhanced facial computed tomography (CT) showed diffuse gaseous necrosis and cutaneous fistula of the upper lip (Fig. 1B). A chest CT performed for persistent dyspnea showed an abscess-like lung
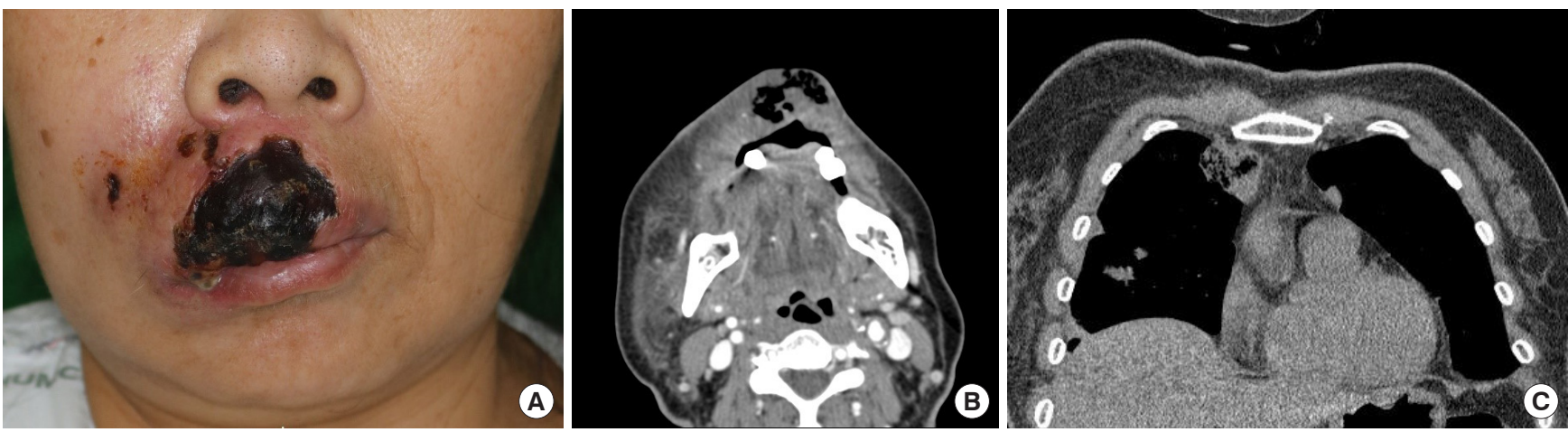

Fig. 1. Initial features of the patient. (A) A 60-year-old woman with diabetes mellitus and gangrenous changes in the upper lip with severe infection. (B) Contrast-enhanced facial computed tomography image showing diffuse gaseous necrosis and cutaneous fistula involving the upper lip. (C) Chest computed tomography image showing an abscess-like lung nodule in the upper right lobe, suspected to be septic emboli, which disseminated from the upper lip infection.
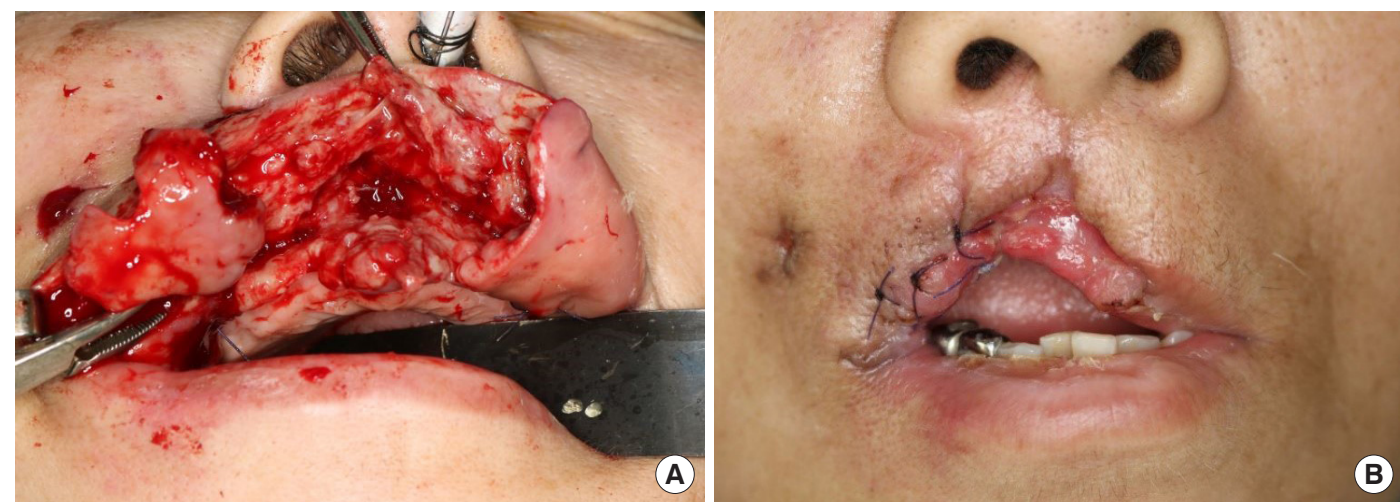

Fig. 2. Several debridements under general anesthesia were performed. (A) Intraoperative view showing full-layer necrosis of upper lip including orbicularis oris muscle. (B) After serial debridement, almost half of the entire upper lip was affected with a full-layer skin defect with scar contracture. 

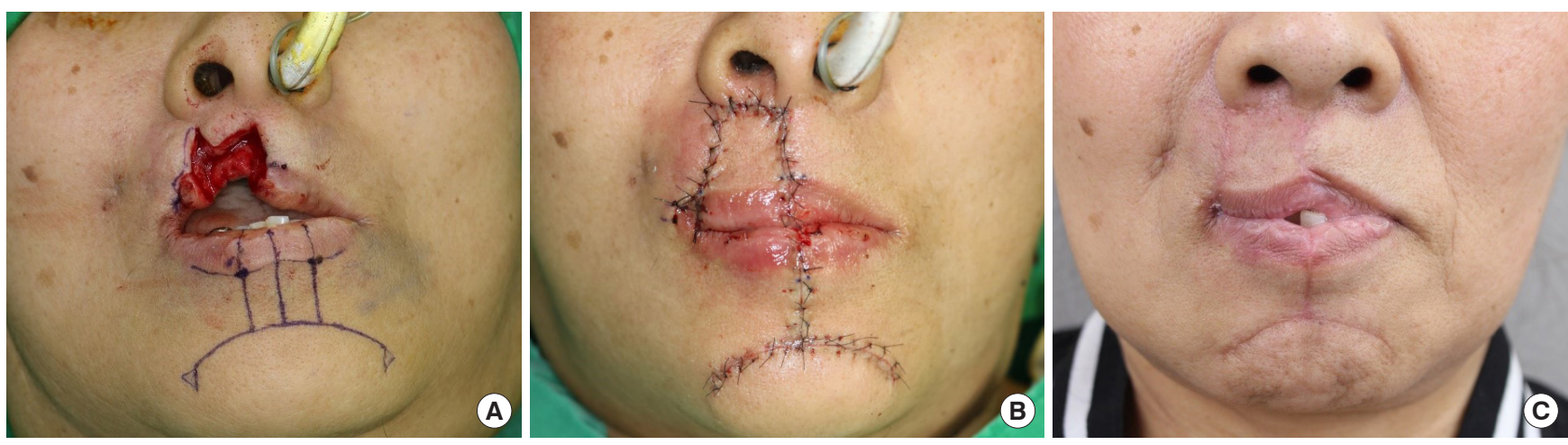

Fig. 3. To correct the drooling and lip seal due to the resultant upper lip defect and scar contracture, scar release and Abbe flap coverage were planned. (A) Six months later, Abbe flap surgery was performed for delayed reconstruction of the upper lip. (B) Immediate postoperative view. (C) Three-month follow-up view.

nodule in the right upper lobe, which may have resulted from septic emboli originating from the upper lip infection (Fig. 1C). The Laboratory Risk Indicator for Necrotizing Fasciitis score was 10 points, placing the patient in the high-risk category; this measure considers C-reactive protein level, white blood cell count, serum hemoglobin, glucose, sodium, and creatinine level. Due to an impression of septic shock as a result of the upper lip NF, immediate surgical intervention was planned. Debridement under general anesthesia confirmed full-layer necrosis of the upper lip including the orbicularis oris muscle (Fig. 2A).

On the 5th day of admission, K. pneumoniae was confirmed in both blood and initial pus cultures; hence, an antibiotic regimen consisting of ceftriaxone and metronidazole was chosen. Serial debridement was performed until the purulent discharge ceased. On the 13th day of admission, the follow-up pus culture confirmed K. pneumoniae and methicillin-resistant Staphylococcus aureus (MRSA). Five weeks after the initial debridement, a full-layer skin and soft tissue defect with scar contracture was evident over almost half of the entire upper lip (Fig. 2B). After thorough upper lip wound care and continuous stabilization for inadequate renal and cardiopulmonary functions and diabetes, she was discharged on the 50th day of admission.

Six months later, a scar release and Abbe flap coverage were performed, with functional and aesthetic considerations, to correct the drooling and lip seal due to the resultant upper lip defect and scar contracture (Fig. 3A and B). Flap detachment was performed after 3 weeks and flap division after 3 months. Follow-up revealed favorable correction of the upper lip drooling. The patient was satisfied with the results (Fig. 3C).

\section{DISCUSSION}

The outcome for general NF patients can be fatal with a mortality rate as high as $29.7 \%$, according to previous studies $[8,10$ -
13]. Therefore, a presumptive diagnosis and rapid treatment are necessary, as delayed diagnosis of NF is directly associated with increased morbidity and mortality. Nonetheless, as seen in this case, early features of NF can easily be mistaken for cellulitis, and thus, require a high index of suspicion. Magnetic resonance imaging and CT play important roles in the diagnosis, differentiating it from cellulitis $[3,14]$. NF should be suspected in patients with rapid progression of infection, abnormal gas accumulation in the soft tissues, and systemic toxicity, especially in those with risk factors such as diabetes, severe kidney, heart or lung diseases, human immunodeficiency virus infection, vascular insufficiency, malnutrition and obesity $[1,3,7,15]$.

Management of NF is based on several principles. The initial surgical excision and debridement help in arresting the spread of the necrotization, stopping the release of inflammatory mediators responsible for systemic complications. A delay in performing aggressive surgery is associated with a high mortality rate. Using antibiotics is the mainstay of antimicrobial treatment. Antimicrobial coverage for MRSA may be needed if this organism is detected or suspected. Daily wound care is essential after surgical debridement, for further removal of compromised tissue. Wound irrigation and wet-to-dry dressing changes are recommended. Reconstruction should be delayed until infection resolves. Adjunctive therapies such as hyperbaric oxygen therapy may be necessary. Hyperbaric oxygen increases free radicals and helps neutrophil-mediated killing of bacteria. It also acts as a bactericide for certain anaerobes. It reduces mortality, shortens the duration of hospitalization and decreases the required number of surgical debridement [3].

Though the most common pathogen contributing to NF is GAS, $K$. pneumoniae is an emerging pathogen around the East Asian region. According to Cheng et al. [9], among immunocompromised NF patients such as diabetics, $K$. pneumoniae is a more common cause than GAS, specifically in Korea. The same 
authors also reported that $47 \%$ of $K$. pneumoniae NF patients died due to septic shock while only $19 \%$ of GAS-NF cases were fatal. Similar results were reported by multicenter studies in South Korea, with approximately $20 \%$ mortality in GAS-NF cases compared to $58 \%$ of mortality in K. pneumoniae NF. Moreover, patients with Gram-negative infections were more likely to have chronic alcoholism, intensive care admission, and septic shock [16]. Furthermore, another study reported that NF patients with DM had more than a seven-fold increase in mortality if $K$. pneumoniae was present in blood cultures and wounds $[8,16]$.

Many cases of K. pneumoniae infection have mixed pathogens, which are more likely to develop bacteremia and distant abscesses. In a report by Jin et al. [17], lower lip NF patients with uncontrolled DM had K. pneumoniae and Candida albicans in tissue culture. In the case presented by Ku and Park [18], a mid-face NF patient with uncontrolled DM had a wound infected by K. pneumoniae and group B streptococcus. Choi et al. [19] also reported a case of mixed infection by K. pneumoniae, Enterococcus faecalis, and Candida species in a flank NF patient with DM. In this case, however, K. pneumoniae appeared alone in the initial culture, and MRSA appeared as a mixed pathogen afterwards. If anaerobic and fungal cultures had been performed initially, mixed pathogens may have been reported in the initial culture. The features reported in the aforementioned cases align with the findings of this study, including underlying DM, mixed pathogens, and rapid progression. Differing aspects in this case, however, were in the manifestation of severe systemic features such as bacteremia, focal septic embolism to the lung, and septic shock due to K. pneumoniae NF. Initial symptoms were minor, resembling those of cellulitis, but within 24 hours, she rapidly deteriorated; thus, this case carried a high risk of mortality.

The essential details highlighted in this case show that if NF is suspected in patients with underlying diseases such as DM, $K$. pneumoniae should be considered as a contributing pathogen, particularly if accompanied by distant dissemination, positive blood culture for K. pneumoniae, and confirmed bacteremia. These features forewarn the very high risk of mortality, and active and intensive care with aggressive debridement and broadspectrum antibiotics should be administered.

\section{NOTES}

\section{Conflict of interest}

No potential conflict of interest relevant to this article was reported.

\section{Ethical approval}

The study was approved by the Institutional Review Board of Kangdong Sacred Heart Hospital (IRB No. 2019-11-004) and performed in accordance with the principles of the Declaration of Helsinki. Written informed consent was obtained.

\section{Patient consent}

The patient provided written informed consent for the publication and the use of her images.

\section{ORCID}

Hyeong Seop Kim https://orcid.org/0000-0003-4037-2805

Yong Joon Chang https://orcid.org/0000-0003-4966-6543

Chul Hoon Chung https://orcid.org/0000-0003-3722-1838

\section{REFERENCES}

1. Hohlweg-Majert B, Weyer N, Metzger MC, Schon R. Cervicofacial necrotizing fasciitis. Diabetes Res Clin Pract 2006;72:2068.

2. Wong CH, Chang HC, Pasupathy S, Khin LW, Tan JL, Low CO. Necrotizing fasciitis: clinical presentation, microbiology, and determinants of mortality. J Bone Joint Surg Am 2003;85: 1454-60.

3. Oguz H, Yilmaz MS. Diagnosis and management of necrotizing fasciitis of the head and neck. Curr Infect Dis Rep 2012;14: 161-5.

4. Shindo ML, Nalbone VP, Dougherty WR. Necrotizing fasciitis of the face. Laryngoscope 1997;107:1071-9.

5. Juncar M, Bran S, Juncar RI, Baciut MF, Baciut G, Onisor-Gligor F. Odontogenic cervical necrotizing fasciitis, etiological aspects. Niger J Clin Pract 2016;19:391-6.

6. Thakur JS, Verma N, Thakur A, Sharma DR, Mohindroo NK. Necrotizing cervical fasciitis: prognosis based on a new grading system. Ear Nose Throat J 2013;92:149-52.

7. Inan CH, Yener HM, Yilmaz M, Gozen ED, Erdur ZB, Oroglu $\mathrm{B}$, et al. Cervical necrotizing fasciitis of odontogenic origin and hyperbaric oxygen therapy. J Craniofac Surg 2017;28:e691-2.

8. Cheng NC, Tai HC, Chang SC, Chang CH, Lai HS. Necrotizing fasciitis in patients with diabetes mellitus: clinical characteristics and risk factors for mortality. BMC Infect Dis 2015;15: 417.

9. Cheng NC, Yu YC, Tai HC, Hsueh PR, Chang SC, Lai SY, et al. Recent trend of necrotizing fasciitis in Taiwan: focus on monomicrobial Klebsiella pneumoniae necrotizing fasciitis. Clin Infect Dis 2012;55:930-9.

10. Golger A, Ching S, Goldsmith CH, Pennie RA, Bain JR. Mortality in patients with necrotizing fasciitis. Plast Reconstr Surg 
2007;119:1803-7.

11. Faucher LD, Morris SE, Edelman LS, Saffle JR. Burn center management of necrotizing soft-tissue surgical infections in unburned patients. Am J Surg 2001;182:563-9.

12. McHenry CR, Piotrowski JJ, Petrinic D, Malangoni MA. Determinants of mortality for necrotizing soft-tissue infections. Ann Surg 1995;221:558-63.

13. Childers BJ, Potyondy LD, Nachreiner R, Rogers FR, Childers ER, Oberg KC, et al. Necrotizing fasciitis: a fourteen-year retrospective study of 163 consecutive patients. Am Surg 2002;68: 109-16.

14. Lin C, Yeh FL, Lin JT, Ma H, Hwang CH, Shen BH, et al. Necrotizing fasciitis of the head and neck: an analysis of 47 cases. Plast Reconstr Surg 2001;107:1684-93.

15. Lee JW, Immerman SB, Morris LG. Techniques for early diag- nosis and management of cervicofacial necrotising fasciitis. J Laryngol Otol 2010;124:759-64.

16. Kim T, Park SY, Kwak YG, Jung J, Kim MC, Choi SH, et al. Etiology, characteristics, and outcomes of community-onset necrotizing fasciitis in Korea: a multicenter study. PLoS One 2019; 14:e0218668.

17. Jin HB, Yang JY, Kim KS, Kim SH, Choe J, Chung JH. Staged lower lip reconstruction following gangrenous stomatitis in an immunosuppressed patient. Arch Craniofac Surg 2018;19:222-6.

18. Ku I, Park JU. Necrotizing fasciitis arisen from nose. Arch Craniofac Surg 2019;20:279-80.

19. Choi J, Cho JT, Choi JY, Jung SN, Seo BF. Necrotizing fasciitis from a percutaneous transhepatic biliary drainage site. J Wound Manag Res 2019;15:130-3. 